\title{
An Enhanced on Bidirectional LI-FI Attocell Access Point Slicing and Virtualization using Das2 Conspire
}

\author{
Yerrolla Chanti, Kothanda Raman, K. Seenanaik, Dandugudum Mahesh, B.Bhaskar
}

\begin{abstract}
LiFi attocell get to systems will be conveyed wherever to help different applications and administration provisioning to different end-clients. The LiFi foundation suppliers should offer LiFi passages (APs) assets as an administration. This, be that as it may, requires an exploration test to be fathomed to progressively and adequately allot assets amonspecialist co-ops (SPs), while ensuring execution detachment among them and their separate clients. This paper presents an autonomic asset cutting (virtualization) conspire, which acknowledges autonomic administration and setup of virtual APs, in a LiFi Attocell get to arrange, in light of SP's and their clients benefit necessities. The proposed asset cutting plan gathers and breaks down the movement insights of the distinctive applications upheld on the cuts characterized in each LiFi AP and appropriates the accessible assets reasonably and relatively among them. It utilizes a control calculation to change the base conflict window of client gadgets to accomplish the objective throughput and guarantee broadcast appointment decency among SP's and their clients.
\end{abstract}

Keywords - LiFi AP, LiFi attocell systems, asset cutting, MAC access virtualization, hypervisor, LiFi activity, VLC, SDN.

\section{INTRODUCTION}

Internet service providers (ISP's) mean to stretch out the fast web to various and testing places. Service providers (SPs) intend to give solid administrations anyplace. These cover clients at cafés, shopping centers, show roads, business and private structures, remote areas, airplane terminals, underground metro and submarines. The LiFi optical correspondence get to technology [1] can meet both SPs and ISPs goals by transforming any light into a remote access point (AP) to empower clients to impart and transmit information at optical (light) speed. The LiFi APs can be arranged through the current lighting systems, or some other rapid optical transport systems, to shape LiFi attocell get to systems. Along these lines, the developing LiFi optical remote access innovation has made a novel web and remote correspondence benefit framework, which gives monstrous

Revised Manuscript Received on July 10, 2019.

Yerrolla Chanti, Department of Computer Science and Engineering, S R Engineering College (Autonomous) Warangal, Telangana India. (E-mail: chanti_y@ srecwarangal.ac.in)

Dr. KothandaRaman, Department of Computer Science and Engineering, S R Engineering College (Autonomous) Warangal, Telangana India. (E-mail: kothanda_raman_d@srecwarangal.ac.in)

Dr. K. Seenanaik, Department of Computer Science and Engineering, S R Engineering College (Autonomous) Warangal, Telangana India. (Email: seena_naik@ srecwarangal.ac.in)

Dandugudum Mahesh, Department of Computer Science and Engineering, S R Engineering College (Autonomous) Warangal, Telangana India. (E-mail: mahesh.dandugudum@ gmail.com)

B.Bhaskar, Department of Computer Science and Engineering, S R Engineering College (Autonomous) Warangal, Telangana India. (E-mail: bhaskar_b@srecwranagal.ac.in) remote system assets [1]. Be that as it may, these ought to be overseen adequately by ISPs and SPs to help the uncommon development and different prerequisites. A utilization case on indoor LiFi attocell Slice cutting. Of remote information and the

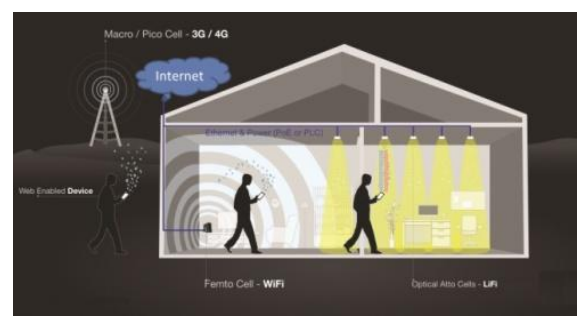

Figure 1. LiFi Attocell using light waves to carry data by Access point slicing

Remote applications. The software-defined networking (SDN) empowered and virtualized [2]. LiFi attocell APs encourage the treatment of reasonable LiFi get to arrange structures for asset deliberation, programmability and separation. These speak to a feasible method to create sufficient instruments for asset administration and bolster novel administrations utilizing separate $\mathrm{LiFi} \mathrm{AP}$ cuts, as appeared in Figure 1.A cut can be characterized as an arrangement of assets which can be resized and customized to help practical administrations to SPs and their clients. For instance, Table I portrays a virtualized LiFi attocell organize comprising of $\mathrm{N} \mathrm{LiFi} \mathrm{APs,} \mathrm{in} \mathrm{which} \mathrm{each} \mathrm{can} \mathrm{powerfully}$ bolster $\mathrm{M}$ cuts at most. A cut includes an arrangement of physical asset squares (RBs) and some cushion space, which can be progressively resized. The distinctive 5G institutionalization bodies take a shot at characterizing the necessities and designs that will empower arrange cutting in $5 \mathrm{G}$ portable access systems.

\subsection{Literature Review}

The general accord is to characterize arrange cut writes and $5 \mathrm{G}$ benefit characterization. A $5 \mathrm{G}$ administration may fall into three categories:

i. Extraordinary portable broadband (x MBB) which requires both high information rates and low inertness in a few territories, and solid broadband over substantial zones. 


\section{AN ENHANCED ON BIDIRECTIONAL LI-FI ATTOCELL ACCESS POINT SLICING AND VIRTUALIZATION USING DAS2 CONSPIRE}

ii. Monstrous machine-type correspondence (m MTC), which needs remote availability for gigantic sending gadgets.

iii. Ultra-dependable and low-dormancy interchanges (u RLLC) or ultra-solid MTC, which covers all administrations requiring ultra-low inactivity connections with a specific level of unwavering quality. The LiFi attocell innovation indicates both the physical layer and the medium access control (MAC) conventions.

The physical layer adjusts the balance and coding plan (MCS) level in view of the channel condition, motion $t$ oimpedance in addition to clamor proportion (SINR), of every client gadget (UD). to enhance the accessible range proficiency. The MAC layer circulates the accessible range among the remote UD's utilizing a multi-client get to convention [3].

Table 1: Life Ap Slices.

\begin{tabular}{|l|l|l|}
\hline AP & Slice spectrum (RBs) & Slice buffer $($ bytes) \\
\hline & $\mathrm{S} 1, \mathrm{~S} 2, \ldots \ldots \mathrm{sm}$ & $\mathrm{B} 1, \mathrm{~B} 2, \ldots \ldots \ldots \mathrm{Bm}$ \\
\hline AP1 & $24,15, \ldots \ldots .40$ & $70,250, \ldots \ldots \ldots .200$ \\
\hline & & \\
\hline APN & $28,28, \ldots \ldots \ldots .35$ & $65,180, \ldots \ldots \ldots, 400$ \\
\hline
\end{tabular}

This timetables the entrance to the LiFi air interface among the UDs utilizing the time as well as recurrence assets in downlink and uplink headings. A SDN work decouples the control plane of the LiFi air interface from its information plane. The control plane determines the planning rationale and administration provisioning approach, though the information plane applies the booking rationale and the movement preparing and dealing with arrangements.

A virtualization layer between these control and information planes gives the virtualization work parameters of each cut designed in a virtualized LiFi AP for the control plane. The SDN work controls the distinctive cuts and projects their asset portion through designing their virtualization work parameters. In this manner, the SDN and virtualization capacities can empower full virtualization for the air interface of a LiFi AP.

A two-advance orthogonal frequency-division multiple access (OFDMA) scheduler can be sent in each LiFi AP to distribute the downlink assets of $\mathrm{LiFi}$ air interface among the bolstered cuts per outline. The virtualization of uplink air interface of LiFi APs depends on the idea of the uplink MAC convention. For instance, the uplink cuts can be segregated by sending a time division multiple access (TDMA) MAC convention, which allocates a restrictive timeshare for each cut in light of its prerequisites. This, be that as it may, may bring about under-using the saved timeshare of a cut since some of them might be left unused within the sight of latent clients of each cut [4].

An arbitrary access convention, in any case, for example, transporter sense different access with impact shirking (CSMA/CA) has a more unique and pioneering nature. It can adaptively deal with the timeshare of each cut by the quantity of dynamic clients. A half and half TDMA/CSMACA MAC convention can conquer the usage wastefulness of the TDMA part, while amplifying the normal aggregate throughput through the CSMA/CA part, in view of its shrewd nature. The downlink range can be overseen among the SPs and their individual UDs at the AP level. Be that as it may, the uplink range can be overseen at the UDs level through controlling their uplink get to channel likelihood and congestion window $(\mathrm{CW})$. For instance, a SPi can acquire a twofold throughput of SPj, if the SPj is allotted a twofold $\mathrm{CW}$ of SPi. The uplink scheduler calculation powerfully saves a variable number of availabilities in each TDMA-CSMA/CA super frame in light of the activity request of clients and required asset reservation of their cut. The unused schedule vacancies in super frames are distributed to unscheduled clients in a dispute based access mode utilizing CSMA/CA convention [5].

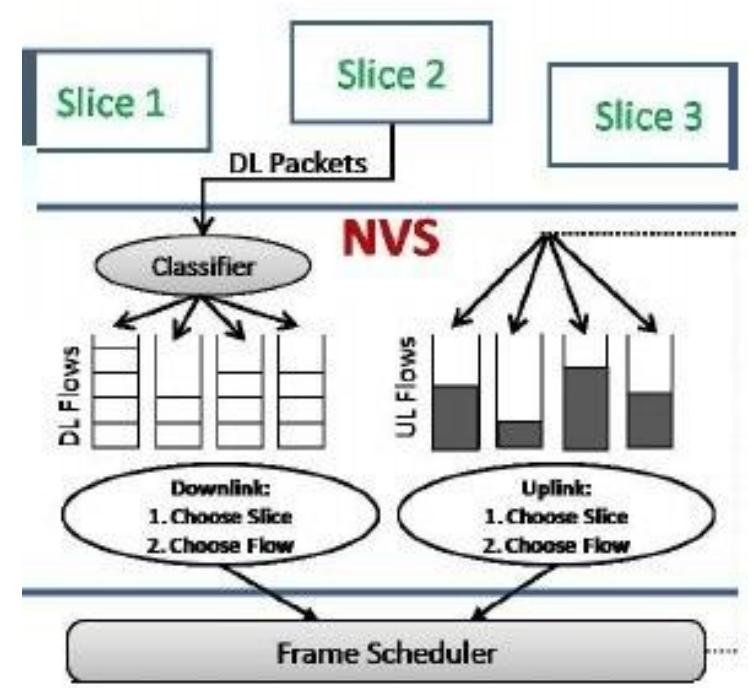

Figure 2. A changed fundamental plan of LiFi AP attocell virtualization

Whatever is left of this paper is sorted out as takes after. In the exploration issue is expressed while elucidating the contributions of this paper contrasted with the primary significant related work. In the LiFi AP virtualization demonstrates is portrayed. In the downlink range cutting and MAC virtualization approaches are talked about. In the uplink range virtualization is clarified. In the SDN application to a virtualized LiFi get to arrange is presented. In the acknowledgment unpredictability of the proposed DAS2 conspire is clarified. In the recreation comes about are displayed. In the conclusions and next research steps are talked about.

\section{PROBLEM STATEMENT AND RELATED WORK}

The LiFi attocell channel attributes are unique in relation to those of a commonplace RF channel. The LiFi channel pick up may not vary when the client is stationary. In any case, the scope of a LiFi AP flag is littler and the UDs may encounter flag deterrents, as they fall all through the FOV (field-of-see) of transmitters/recipients.

Published By: 
While, stationary LiFi UDs for the most part encounter pretty much steady throughput, the UDs with versatility and nonstop introduction varieties may encounter a drop in their throughput. Along these lines, the test is the way to accomplish a viable and reasonable cutting of LiFi attocell assets, autonomous of limit fluctuation and accessibility of LiFi AP assets. Additionally, each 5G administration may have diverse necessities regarding inertness, transfer speed, security and d A basic asset portion approach parcels the spectrum band into various sub-groups and allocates each SP administrator a sub-band for all time (i. e a settled arrangement of asset units).

This devoted asset sharing model isolates the control and client movement of the diverse cuts, which reduces cut flexibility and in addition adaptability, and limits the asset multiplexing pick up. Hence, this model does not permit intraentomb administrator asset getting. Then again, a hypervisor can be sent over the LiFi MAC and physical assets to progressively dispense assets to virtual administrators as per their movement requests and clients' channel conditions.

Table 2 condenses the primary created ways to deal with help remote access systems cutting and virtualization. The idea of system virtualization substrate (NVS) is presented in [6], as appeared in Figure 2. It proposes a cut scheduler, which progressively allots MAC layer assets to cuts with the goal that each cut (administrator) can accomplish its saved physical asset units or ensured throughput rate. In the creators stretch out (NVS) to help incomplete asset reservation utilizing a two-advance confirmation control plot. This saves a base asset for every administrator and offers the rest of the range among the clients of the diverse cuts following the primary start things out served planning discipline.

However, the proposed cutting scheduler and twoadvance confirmation control approach can't be sent to help a self-assertive number of cuts. Notwithstanding the established portable broadband application benefits, the proposed virtual design should bolster some other vertical industry applications. These may incorporate car frameworks, keen matrices, and shrewd city and IoT administrations. A MAC convention is intended to referee medium access among numerous clients, yet it ought to be upgraded to help a variable number of cuts in light of asset sharing correction. This paper presents a coordinated asset virtualization structure for vitalizing $\mathrm{LiFi}$ attocell AP assets to sup-port a dynamic number of cuts [7].

An autonomic virtualization conspire is created to help autonomic downlink and uplink asset assignment and sharing among the distinctive cuts in light of their activity loads, proprietors need and expert versioned benefit prerequisites. The created dynamic LiFi AP range sharing (DAS2) conspire keeps running crosswise over $\mathrm{LiFi}$ physical/MAC layers. It powerfully shares uplink and downlink assets in light of data got from the physical, MAC and application layers, following a cross-layers configuration show. This empowers UDs to get administrations from their cuts in view of downlink range units control and broadcast appointment control that guarantees benefit confinement and decency among UDs inside and crosswise over cuts. The proposed asset virtualization outline work has been widely assessed to exhibit the fundamental a subjective number of cuts. A broadcast appointment control deliberation work chooses a particular physical transmission rate for each bolstered cut, while ensuring a base administration rate for dynamic UDs.

It progressively assigns assets to the distinctive SPs in view of their activity stack and the accessibility of LiFi AP downlink and uplink assets. The downlink scheduler can bolster various administration classes on each cut characterized in a LiFi AP. It is related with a confirmation control to ensure the QoS of dynamic cuts. The execution comes about show the thoroughness, adaptability and versatility of the proposed cutting plan in sharing assets among cuts characterized in a LiFi AP.

\subsection{System Model}

The unmistakable light correspondence (VLC) downlink and infrared (IR) uplink range, and in addition the cradle of a LiFi AP can be progressively sorted out into cuts in Decker Containers (DC's), as appeared in Figure 2. Every DC is portrayed by its dispensed range limit and cradle space, as clarified in Table 1. A DC can act naturally contained with its asset scheduler and MAC convention to serve its bought in UDs following a dynamic sharing asset approach. The hypervisor utilizes the DC (virtual machine (VM)) screen/director to screen man-age and progressively organize access to physical and MAC assets among upheld cuts. This keeps up detachment among physical assets designated to dynamic cuts (DCs) per downlink and uplink MAC outlines.

It additionally permits asset getting among cuts. The AP controller parleys the entrance and control activity admission to the physical assets, which are apportioned in DCs. We expect that the LiFi AP utilizes physical layer culminate channel state data (CSI) and a versatile balance and coding (AMC) conspire. These adapt to any debasement in channel quality to boost the range throughput. OFDMA and TDMA-CSMA/CA MAC conventions are sent to help various clients' access in LiFi downlink and uplink headings, separately. A UD is furnished with a remote handset that backings the VLC range in downlink and IR in uplink headings and IEEE $802.11 \mathrm{Wi}-\mathrm{Fi}$.

The UDs just convey through Wi-Fi when they are out of the LiFi AP scope or experience light channel blocking. The downlink range is evaluated in recurrence and time spaces regarding least range units called RBs and got to in schedule openings. A solitary RB may comprise of one or different subcarriers as per the remote innovation. The aggregate number of subcarriers accessible on the LiFi downlink can be computed by increasing the quantity of RBs, $N_{S C}^{R B}$, by the quantity of subcarriers per RB, N RB. In this way, the data transfer capacity of a solitary subcarrier can be figured by separating the aggregate reasonable $\mathrm{LiFi}$ downlink bandwidth, by the quantity of advantages of virtualization and analyze the execution of a downlink range subcarriers, for example, 


\section{AN ENHANCED ON BIDIRECTIONAL LI-FI ATTOCELL ACCESS POINT SLICING AND VIRTUALIZATION}

USING DAS2 CONSPIRE

$$
b_{S C}=\frac{B}{N_{R B}^{D L} \times N_{S C}^{R B}}
$$

a virtualized LiFi AP to a non-virtualized LiFi AP. Rather than the cutting edge work over, the proposed virtualization conspire upholds dynamic sharing of LiFi down-connection and uplink assets cuts among SPs and their separate client gatherings. It runs in light of straightforward reflections for the MAC and physical assets of LiFi APs to dependably bolster.

The UDs just convey through Wi-Fi when they are out of the LiFi AP scope or experience light channel blocking. The downlink range is evaluated in recurrence and time spaces regarding least range units called RBs and got to in schedule openings. A solitary RB may comprise of one or different subcarriers as per the remote innovation. The aggregate number of subcarriers accessible on the LiFi downlink can be computed by increasing the quantity of RBs, NDL, by the quantity of the data transfer capacity of a subcarrier data transfer capacity is thought to be little, contrasted with the intelligent remote channel transmission capacity. The LiFi downlink bandwidth, "B" Hz, and the support space, "Q", are progressively shared among an arrangement of cuts. A SP is distributed a cut which can be shared among its clients. A downlink virtual demand, $r$, is spoken to by (ID, $\mathrm{p}, N_{R B}, \mathrm{t}_{\mathrm{s}}$ ), where ID dependability, which requires a suitable method to give a system cut customized to each administration. Speaks to the cut identifier which demonstrates the cut kind (e.g. which scheduler) notwithstanding the inhabitant ID who is responsible for the cut, $\mathrm{p}$ speaks to the need level, $N_{R B}$ speaks to the base number of asked for RBs, and ts speaks to the base number of required vacancies. The time access to the IR uplink range of LiFi APs is separated into settled length half and half edges.

Table 2: Wireless Net Works Resource Slicing And Virtualization Related Work

\begin{tabular}{|l|l|l|l|l|}
\hline Technology & objective & Uplink & Downlink & $\begin{array}{l}\text { Proof of } \\
\text { concept }\end{array}$ \\
\hline $\begin{array}{l}\text { WiMax } \\
{[16]}\end{array}$ & $\begin{array}{l}\text { slicing } \\
\text { framework }\end{array}$ & $\begin{array}{l}\text { slice and } \\
\text { flow } \\
\text { scheduling }\end{array}$ & $\begin{array}{l}\text { slice and } \\
\text { flow } \\
\text { scheduling }\end{array}$ & NVS \\
\hline LTE[17] & $\begin{array}{l}\text { resources } \\
\text { assignment- } \\
\text { based } \\
\text { predefined } \\
\text { load } \\
\text { balancing } \\
\text { levels }\end{array}$ & NO & $\begin{array}{l}\text { downlink } \\
\text { PRBs } \\
\text { scheduler }\end{array}$ & NO \\
\hline $\begin{array}{l}\text { IEEE } \\
\text { 802.16 } \\
\text { (WiMax }) \\
{[18],[19]}\end{array}$ & $\begin{array}{l}\text { Air-Tim } \\
\text { fairness and } \\
\text { slice } \\
\text { isolation }\end{array}$ & NO & $\begin{array}{l}\text { traffic } \\
\text { shaping }\end{array}$ & $\begin{array}{l}\text { WiMax } \\
\text { Prototype }\end{array}$ \\
\hline $\begin{array}{l}\text { IEEE } \\
\text { 802.11 }\end{array}$ & $\begin{array}{l}\text { efficient } \\
\text { experiments } \\
\text { isolation on }\end{array}$ & $\begin{array}{l}\text { TDM- } \\
\text { based slice }\end{array}$ & $\begin{array}{l}\text { TDM-based } \\
\text { slice }\end{array}$ & ORBIT \\
\hline (WiFi) [19] & $\begin{array}{l}\text { lexperimental } \\
\text { wireless } \\
\text { tested }\end{array}$ & Scheduling & Scheduling & $\begin{array}{l}\text { Software } \\
\text { interface }\end{array}$ \\
\hline IEEE & TDMA- & NO & airtime & NO \\
\hline
\end{tabular}

\begin{tabular}{|c|c|c|c|c|}
\hline 802.11 [14] & based slicing & & $\begin{array}{l}\text { based } \\
\text { slicing }\end{array}$ & \\
\hline $\begin{array}{l}\text { IEEE } \\
802.11 \text { [11] }\end{array}$ & $\begin{array}{l}\text { Uplink } \\
\text { slicing }\end{array}$ & $\begin{array}{l}\text { airtime- } \\
\text { based } \\
\text { slicing }\end{array}$ & $\mathrm{NO}$ & Split AP \\
\hline $\begin{array}{l}\text { LTE RAN } \\
{[15]}\end{array}$ & $\begin{array}{l}\text { LTE RAN } \\
\text { sharing }\end{array}$ & NO & $\begin{array}{l}\text { partial } \\
\text { sharing }\end{array}$ & $\mathrm{NO}$ \\
\hline
\end{tabular}

A mixture outline comprises of three stages: head (synchronization) transmission, TDMA stage and CSMA/CA stage, as appeared in Fig. 3 [8]. The leader of each edge has a grouping that synchronizes the transmitter clock of LiFi APs with that of UDs. The schedule openings of the half and half TDMA-CSMA/CA outline are allotted adaptively to the distinctive uplink cuts in view of their administration necessities. High need UDs send activity amid the schedule vacancies which are assigned to the TDMA stage While low need UDs send movement amid the rest of the schedule vacancies distributed to the CSMA/CA stage. In each super frame, $t$, a most extreme number of schedule vacancies, $\mathrm{S} \max (\mathrm{t})$, are progressively designated to the TDMA stage in light of the aggregate high need movement and Q o $S$ required for each cut.

The CSMA/CA convention keeps running with each vacancy partitioned into its back-off time units. The MAC controller screens the normal support level, qt $k$, of UD k, $\forall \mathrm{k} \in \mathrm{K}$, toward the start of super frame. It keeps up the normal support level data for UD $\mathrm{k}$ in the shape (qt $k, \mathrm{ftk})$, where ftk is the quantity of super frames which have gone after the most recent report was gotten; and $q k\left(t^{\prime}-1\right)$ is the cradle level of UD $k$ got in the most recent report. The MAC controller gauges the normal cradle level of UD $\mathrm{k}$ as takes after:

$$
q^{\prime} k(\mathrm{t})=q^{\prime} k(\mathrm{t}-1)+\left\lfloor\lambda_{t k} f_{t k}\right\rfloor
$$

Where $\lambda_{t k}$ indicates the mean entry rate of movement produced by UD $\mathrm{k}$ per super frame $\mathrm{t}$. The cushion status of $\mathrm{UD} \mathrm{k}$ is resolved as, Btk $=q^{\prime} k(\mathrm{t})$, if $q^{\prime} k(\mathrm{t})<\mathrm{Bmax}$; generally $\mathrm{Btk}=\mathrm{B} \max . q^{\prime} k(\mathrm{t})$ May be higher than $\mathrm{B} \max$ [9]. It sorts the UDs in rising request of their cushion levels. A downlink or an uplink stream ask for is conceded into a cut if it's asked for assets are effectively apportioned. The conceded stream holds its distributed assets until the point when it stops.

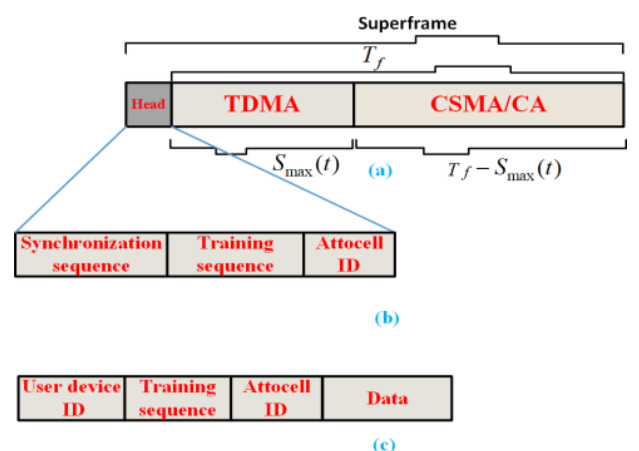

Figure 3. Frame structure in LiFi system.

Published By:

Blue Eyes Intelligence Engineering

\& Sciences Publication 
(a) Hybrid uplink outline structure (b) Attocell information outlines structure. (c) Feedback information outlines structure.

The stream demand can be conceded amid a given schedule vacancy, it stays in the cradle to retry confirmation in whenever opening, if conceivable. At the point when the stream ask for isn't effectively conceded after various availabilities, it is expelled from the cushion and the stream ask for is rejected. The most extreme postponement can be distinctive for each need cut demand level. The LiFi AP keeps up a mapping section for each cut ID and its comparing IP address, which is imparted by the cut orchestrator amid the cutting instantiation process. The clients of the diverse cuts are related with their virtualized LiFi AP (cut) through restricting their IP address with a reporter expansion benefit set identifier (ESSID) promoted in the synchronization guides [10].

\subsection{Downlink Resources Slicing}

A slope based OFDMA scheduler dispenses assets to bolstered cuts in each LiFi attocell AP. Downlink activity parcels are policed and ordered in light of their cut ID. Top level scheduler serves the diverse cuts in light of their need weight in climbing request. The unused RBs by the emptied cuts are booked among the over-burden cuts in light of Eq. (2).

This procedure proceeds until the point that the RBs are altogether distributed; or the cuts are altogether served. At the lower level, an upgraded start-time fair queuing (SFQ) scheduler [11] serves parcels sitting tight in the distinctive lines for transmission in the diverse cuts. It plans the RBs among the distinctive clients as indicated by the quantity of RBs distributed to their comparing cuts. Each line is relegated an administration information rate weight in light of its administration need class, c, $\psi_{i c}$ such that, $\sum_{c \in C} \psi_{i c} \Sigma \leq$ 1. A worldwide virtual time, $v_{i}$, tracks the bundle $c \in C$ administration all things considered. Each line has nearby begun and wrap up times, which are indicated by Sic and $F_{i c}$, separately. At the point when another parcel touches base at a vacant line, $\mathrm{c}$, the begin and complete circumstances of the head-of-line (HOL) bundle are refreshed as takes after:

$$
\text { Sic }=\max \{\text { Fic, vi }\} \text {; }
$$

They are mapped into a relating line in the cut

$$
F_{i c}=S_{i c}+\frac{L_{i c}}{\psi_{i c}}
$$

Where, yic in view of their administration class, as appeared in Fig 4. The MAC estimation controller computes, per schedule opening, the aggregate number of conceded movement parcels in A Pi onto cut m, Qtm, $\forall \mathrm{m} \in$ $M$, in view of Eq.(1), as takes after: $\mathrm{K}$ where Lic signifies the parcel length of class $\mathrm{c}$ in APi.

The bundles which touch base at non-void cushions sit tight for their administration. The line with the base HOL begin time is chosen for transmission. On the off chance that the HOL bundle can be transmitted in the doled out schedule opening, without discontinuity, it is De-queued

$$
Q_{t m_{i}}=\sum_{k=1}^{K} B_{t k_{m}}
$$

$k=1$ furthermore, the worldwide virtual time is refreshed to the begin time, i.e., Sij [20]. A short time later, the related neighborhood begin and complete circumstances are The neighborhood DAS2 calculation ensures each cut characterized in an $\mathrm{A} P \mathrm{Pi}$ a base number of RBs, $\mathrm{R}$ min, per vacancy. A cut $m$, which requires various $R B s$ more than $R$ min, is viewed as over-burden and the other way around. In light of Eq. (5), the MAC estimation controller ascertains, per schedule opening, the movement volume vector of cuts characterized in an $\mathrm{AP}_{\mathrm{i}}, \forall \mathrm{i} \in \mathrm{N}$, as takes after:

$$
\mathrm{Qi}(\mathrm{t})=[\mathrm{Qi1}(\mathrm{t}), \mathrm{Qi2}(\mathrm{t}), \ldots, \mathrm{QiM}(\mathrm{t})], \forall \mathrm{i} \in \mathrm{N}, \mathrm{m} \in \mathrm{M} \text {. }
$$

In view of Eq. (6), the downlinks RBs of each AP are refreshed. On the off chance that the line is nonempty, these updates are indistinguishable to the landing case.

$$
w_{\mathfrak{I}}=\frac{Q_{\mathfrak{I}}(t)}{\sum_{m=1}^{M-U} Q_{\mathfrak{I}}(t)}
$$

Something else, the begin time is set to a vast incentive to expel it from conflict, i.e., $S_{i c}=\infty$.

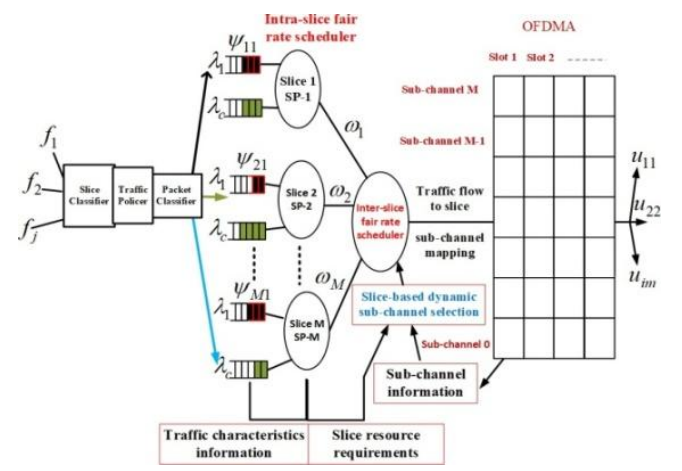

Figure.4. LiFi Downlink spectrum virtualization

\subsection{Slice Benefit Estimation Module:}

This module evaluates the moment data transfer capacity required to transmit the movement stream in line $\mathrm{j}$. An ensured data transmission (GB) stream, $\mathrm{j}$, which is conceded in cut $\mathrm{m}$, has a qualified transfer speed ask for, ri, given as: $\gamma j$ conveyed among the characterized cuts in light of corresponding weights. These are computed as takes after:

$$
r_{e j}=\max \left\{\frac{Y_{j t}}{8}\left[t-S_{j}(t)\right], 0\right\}
$$

Where $\gamma$ jt indicates the objective movement rate (bps) of stream $\mathrm{j}$; and $\mathrm{t}$ is the clock time of LiFi AP.

The administration estimation module keeps up an administration clock for every GB stream running in the cut $\mathrm{I}$; and $\mathrm{Sj}(\mathrm{t})$ is the administration clock estimation of stream $\mathrm{j}$ at time where wim signifies the heaviness of cut $\mathrm{m}$ characterized in $\mathrm{APi}$; and $\mathrm{U}$ indicates the quantity of under loaded cuts. In light of Eq. (4), an over-burden cut $\mathrm{m}$ in APi can be distributed a most extreme RBs, $\mathrm{R}$ maxim, as takes after: $\mathrm{U} t$, which applies the idea of the Virtual Clock [12]. This administration clock is synchronized with the clock of a LiFi AP, when a stream is conceded. The clock ticks with the accompanying an incentive upon the administration of every parcel in its comparing stream: 

USING DAS2 CONSPIRE

$$
R_{\text {max }_{\mathfrak{I}}}=R_{\text {min }_{\mathfrak{I}}}+\left\lfloor\left(N_{i}^{e f f}-\sum_{j=1}^{U} R_{\mathfrak{J}}\right) w_{\mathfrak{I}}\right\rfloor
$$

Where $N_{i}^{e f f}$ indicates the quantity of successful RBs in API; and Rim means the RBs required for the under loaded cut $\mathrm{m}$ in $\mathrm{AP}_{\mathrm{I}}$. In view of Eq. ( 8), the quantity of RBs can be resolved for all the bolstered cuts in every a pus. Each cut is doled out an administration need weight in light of its movement load and sort. At each sub-outline (schedule vacancy), the where $A_{j}$ is the administration clock addition of stream $\mathrm{j}$, upon the administration of bundle with size of $\mathrm{L}_{\mathrm{j}}$ bytes; and $\gamma_{\mathrm{jm}}$ is the estimation rate (in bps) for stream j. For the administration clock, this esteem ought to be the objective rate, $\gamma_{\mathrm{j} t}$. A different variable records the virtual time of stream $j$ as opposed to stamping each arrived parcel with its virtual time, as in [13]. A few parcels might be dropped because of their infringement of greatest dormancy The administration clock edges the administration rate of stream $\mathrm{j}$ inside its objective movement rate.

\subsection{Slice Implementation Module to the QOS:}

This module keeps up a QoS clock for each conceded GB stream. The QoS clock is introduced with the clock of LiFi $\mathrm{AP}$, yet increments with the esteem chosen by the diverse estimation rate characterized in Eq.(10), given as takes after:

$$
A_{j}=\frac{8 . L_{j}}{r_{\min _{j}}}
$$

Where $r_{\min _{j}}$ means the base saved movement rate of stream .The QoS clock implements the administration rate of stream $\mathrm{j}$ to meet an ensured esteem. The QoS authorization module separates the transmission capacity of stream into ensured bandwidth (GB) and non-ensured data transmission (NGB) in view of the estimation of the QoS grouping and attocell ID, as appeared in Figure $3 \mathrm{~b}$. The synchronization is acknowledged when the arrangement design is recognized by UDs. The uplink casing of UDs comprises of UD ID, preparing succession, attocell ID information, as appeared in Figure 3c. After getting the synchronization arrangement, the UD sets its check to transmit in its assigned vacancy. The preparation arrangement empowers the UDs to recognize the SNR and the intensity of got and transmitted signs. This data is utilized to assess the nature of channel conditions. Amid the CSMA/CA stage, the planning synchronization work (TSF) determined by the IEEE 802.11 standard guarantees the synchronization among the fighting low need UDs. The TSF work is utilized for synchronization by occasionally trading data through reference points containing timestamps. The UDs which have low-information rate involve more broadcast appointment, contrasted with the UDs with a higher information rate and a similar bundle estimate [36]. The broadcast appointment, aki , is the part of channel clock, Stj, given as takes after: time get to designated to stream j j created by UD k, which is bought in with cut's SP administrator I in one-moment interim, given as takes after:

$$
\begin{aligned}
& r_{j}^{G B}=\min \left\{r_{j}, \frac{r_{\min _{j}}}{8}\left(t-S_{t j}\right)\right\}, S_{t i}<t ; \\
& r_{j}^{G B}=0, S_{t j} \geq t \\
& r_{j}^{N G B}=r_{j}-r_{j}^{G B}
\end{aligned}
$$

The transfer speed demand of $\mathrm{BE}$ stream is viewed as dependably as NGB. The transfer speed of GB stream can be separated into two where $\gamma$ I signifies the ensured information rate of stream $\mathrm{j}$ created $\mathrm{j}$ by UD $\mathrm{k}$; and ri signifies the physical transmission information rate parts in view of the postponement of its bundles. The transfer speed of a few parcels is ensured in the casing $\mathrm{n}$, and the transmission capacity of UD $\mathrm{k}$ bought in with SP I. A client $\mathrm{k}$ produces a stream of whatever remains of stream bundles is ensured in the edge $n+2$ in view of the aggregate postpone prerequisite of stream j. $j$ with various parcels, $P_{k j}$, is apportioned a broadcast appointment with an ensured information rate, $\gamma_{\mathrm{kj}}$, as takes after:

\subsection{Uplink MAC Access Virtualization}

The uplink mixture TDMA-CSMA/CA MAC convention man-ages MAC get to in light of client and produced movement need.

$$
a_{k_{j}}=\frac{P_{i} L_{k}}{r_{k}}+\mathrm{OH}
$$

The edge spaces are conveyed among the diverse uplink cuts in view of their need movement. The rest of the schedule openings in the casing are allotted to the diverse low need clients as indicated by the conflict procedure characterized in the CSMA/CA convention. A synchronization procedure is required to empower the distinctive UDs to transmit in their schedule openings and maintain a strategic distance from any crash amid the TDMA stage. Where dj and Lkj mean the postpone spending plan and the greatest parcel size of stream $j$, separately; and $\mathrm{OH}$ indicates the overhead time required to transmit the additional control, acknowledgment and synchronization bits alongside the payload of stream $\mathrm{j}$. In view of Eq.( 2), the required broadcast appointment to cut I can be figured as takes after: An entire outline for an indoor ultra-thick VLC arrange is presented in [14], which incorporates a full synchronization process. This is organized at framework and cell levels to alleviate between cell obstruction and dodge medium access impacts in each attocell, individually.

$$
a_{i}=\frac{Q_{t_{l}}^{\prime}}{\sum_{i=1}^{M} Q_{t_{l}}^{\prime}}
$$

The procedure synchronizes where the required broadcast appointment to UD $\mathrm{k}$ can be ascertained as the range allotment with the diverse LiFi APs and the client's access to the transmission medium in each $\mathrm{LiFi}$ cell utilizing TDMA. takes after:

$$
a_{k_{j}}=\frac{q_{t k_{j}}^{\prime}}{Q_{t_{l}}^{\prime}}
$$

The leader of the uplink super frame contains data that empowers the UDs to synchronize with the clock of their LiFi APs, as appeared in Fig. 3a. A LiFi attocell is doled out a special identifier (ID) in light of its AP's position organizes. A known piece design speaking to a synchronization succession is presented toward the start of each TDM outline (i.e. time cycle) to empower UDs to get 
to the medium. The example of the synchronization arrangement may have diverse lengths or has a particular example and same length. The uplink edge of the LiFi AP comprises of three sections: synchronization succession, preparing The UD $\mathrm{k}$ transmits in the CSMA/CA stage with a probability conversely corresponding to its base conflict window ( $\mathrm{C} \mathrm{W}$ min). The all through of UD $\mathrm{k}$ can be estimated in view of the measurable reports gathered each inspecting window of successful parcels transmission. The deliberate throughput of UD, $\mathrm{k}, \gamma \mathrm{km}$, is contrarily corresponding to its doled out $\mathrm{C} \mathrm{W}$ min. A policed is created to change the $\mathrm{C} \mathrm{W}$ min of UDs bought in with the diverse cuts to ensure their relative throughput subject to their cuts' maximal throughput. Generally, the MAC controller calculation screens the deliberate through put clients. The cuts are segregated over each super frame to limit the execution effect of asset designation and use in one cut over the others. A neighborhood bank can acquire from the worldwide bank in light of a need record, wim, which can be computed as: $\omega \mathrm{Em}$. BLm The neighborhood bank can get tokens from the worldwide bank up to a specific credit, $\mathrm{cm}$, which characterizes the most extreme number of obtained tokens. Essentially, a stream $\mathrm{j}$ can acquire tokens from the nearby bank up to a specific credit, $\mathrm{cm} \mathrm{j}$. The credit esteems guarantee decency among applications in obtaining tokens from the worldwide and nearby banks. At the point when the neighborhood bank and a stream have counters, Em and Em $\mathrm{j}=0$, this implies the token age rate rises to the stream information rate amid the casing. The neighborhood bank and the stream may acquire tokens from the worldwide and nearby banks up to a characterized charge constrain. Both the stream and the neighborhood bank should give various tokens with the goal that they can get tokens. As far as possible for a neighborhood bank $\mathrm{dm}$ and, $\mathrm{dmj}$, for a stream $\mathrm{j}$ on cut $\mathrm{m}$ are set to negative qualities, so vindictive nearby bank or stream in a similar cut can't influence the QoS of other all around carried on streams in the cut [15]. The eTBFQ plot misuses the factual multiplexing of conceded streams in cuts to enhance the general data transfer capacity usage of the LiFi uplink range.

\subsection{SDN Controller Function}

The fundamental SDN controller work comprises of checking some basic parameters identifying with bolstered cuts and their clients movement administration in each $\mathrm{LiFi}$ AP. The checking procedure of virtualized LiFi APs is essential in instantiating and arranging downlink and uplink cuts while controlling and overseeing asset allotment in virtualized LiFi attocell APs. A brought together unit (CU) handles input data collected from UDs and also the system state to empower the distinctive VLC cells to get to the range. This plans to help solid multi user access in every cell. In this reference, the created organize design takes after the SDN idea with an essential plan to moderate between cell obstructions and enhance the nature of information transmission on the VLC remote medium. The DAS2 conspire uncovered some down-connection and uplink parameter data to the brought together SDN controller. The parameters per LiFi AP may include: I) cuts use level, ii) number of UDs and their activity stack per cut, iii) channel quality pointer (CQI) or (SNR) of each UD per cut, and iv) inactivity of the most established parcel in the UD's line and its throughput.

The DAS2 conspire utilizes the examination of checked information to concede streams into bolstered cuts per $\mathrm{LiFi}$ AP. It likewise utilizes the data to plan UDs over the virtual RBs relegated to the distinctive cuts. The SDN parameters data which portrays uplink cuts in a $\mathrm{LiFi}$ attocell AP including the e-TBFQ parameters. This incorporates the charge, credit, nearby and worldwide banks counters, and in addition token produce rates characterized per cut in each LiFi AP. The SDN controller can progressively design uplink and downlink parameters to guarantee the QoS prerequisites of utilization bolstered on each cut characterized in LiFi APs.

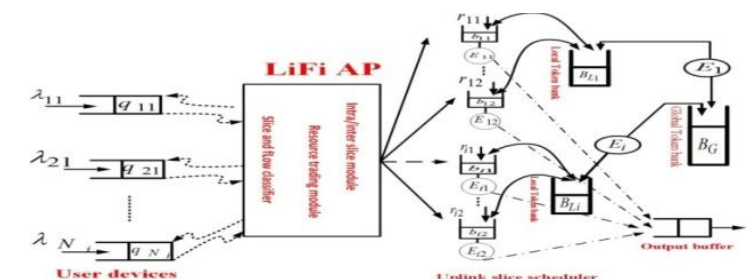

Figure 5. Asset cutting plan pseudo code: Complexity examination

\subsection{SDN Controller Operation:}

The brought together SDN controller keeps up the SDN parameters utilized for cutting by the DAS2 plot in databases. It can refresh the SDN parameters and present (characterize) new ones in light of new applications necessities. These parameters can be utilized to program assets assignment to the distinctive cuts made or instantiated in LiFi attocell APs. The LiFi AP assets are progressively shared and apportioned to the diverse cuts. The SPs give their administrations and application data prerequisites to the SDN controller, which are gotten through the northbound interface. The SDN controller designs the parameters of cuts or instantiates new ones in the applicable APs, which can all the more likely serve them. It imparts this data through the southbound interface to the neighborhood AP's controller. This updates asset distribution to the diverse cuts in LiFi APs, which can meet the QoS prerequisites of clients. It controls and deals with the assets of APs and designs them to adapt to organize elements and meet QoS prerequisites. The worldwide confirmation control component keeps running in the SDN controller to deal with the quantity of cuts arranged in each AP. The cuts (SPs) can actualize their stream level affirmation control to guarantee that streams with basic asset necessities don't endure, and perform elegant debasement in case of asset over-burden.

\subsection{Airtime Abstraction Function:}

The QoS of utilization's which run onto cuts characterized /instantiated in a LiFi attocell AP can be corrupted by various components. These incorporate the quantity of accessible RBs, channel quality conditions and kind of administration regarding deferral or throughput. 


\section{AN ENHANCED ON BIDIRECTIONAL LI-FI ATTOCELL ACCESS POINT SLICING AND VIRTUALIZATION USING DAS2 CONSPIRE}

Confirmation controller instrument ought to be sent in each LiFi AP to confine the affirmation of activity streams in view of the QoS necessities of uses running onto cuts upheld in each LiFi AP. The conceded clients are allowed a maximal adjust of decency and usage of administrations. Another stream with a broadcast appointment share, $\mathrm{a}_{\mathrm{ik}}$, can be confessed to transmit on the LiFi uplink channel subject to the accompanying limitation [16]:

$$
a_{K^{\prime} j}+\sum_{k \varepsilon K} \sum_{j \varepsilon J} a_{k_{j}} \leq E A
$$

Where EA signifies the successful broadcast appointment of the LiFi uplink channel. It is characterized as the most extreme level of accessible broadcast appointment that can be apportioned to UDs; $\mathrm{a}^{\prime} \mathrm{j}$. indicates the broadcast appointment share designated to stream $\mathrm{j}$ produced by UD $\mathrm{k}$. These are found in the rate table kept up in the LiFi driver introduced in the UD, Sending the gathered parameters of UDs in stage 1 to the SDN controller, which ascertains the most extreme broadcast appointment limits for the dynamic UDs and sends them to the nearby AP controller and changing over the greatest broadcast appointment point of confinement of each UD to a transmission rate esteem in view of the accessible assets of LiFi APs. This powers the forming module in each UD to reset the transmitted rate point of confinement to the esteem implemented by the SDN controller. The forming module can be actualized by utilizing the Click particular switch, which straightforwardly controls the outbound movement from the UD interface.

\subsection{Complexity Analysis}

The multifaceted nature examination gives a surmised arrange number to the activities or ventures to make and design cuts in each LiFi AP in an attocell organize, and in addition to ensure the objective QoS prerequisites for the two cuts and their UDs. To improve the unpredictability calculation of the DAS2 conspire, Figure 6 demonstrates the investigation for a cut $\mathrm{LiFi}$ attocell AP. The tasks underlining the DAS2 plot don't include framework vector increase and network reversal. Accordingly, the acknowledgment multifaceted nature of the DAS2 conspire is viewed as low, as clarified in Figure 6

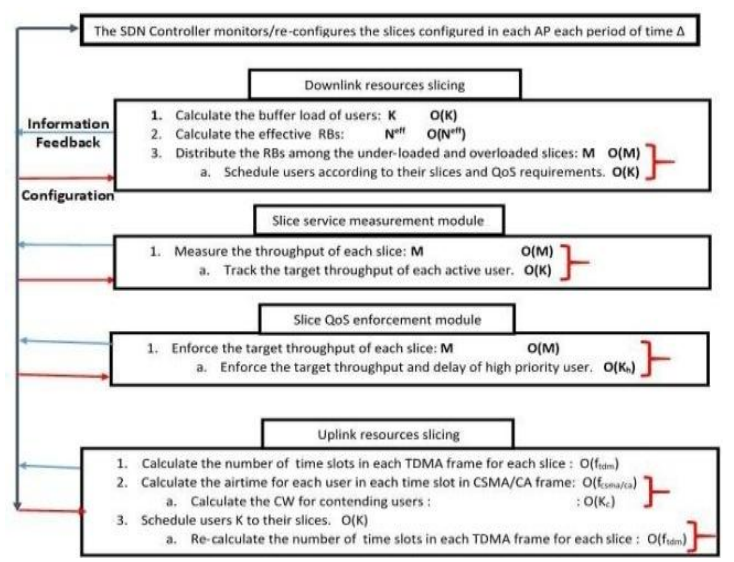

Figure 6. Resource slicing scheme pseudo code: Complexity analysis

The multifaceted nature of cutting downlink assets in a $\mathrm{LiFi}$ attocell $\mathrm{AP}$ requires various tasks of request $\mathrm{O}(\mathrm{MK})$, where $\mathrm{M}$ and $\mathrm{K}$ indicate the quantity of cuts and UDs, individually. A similar number of tasks is required to gauge and authorize the QoS necessities of cuts and UDs in a LiFi attocell AP. It has roughly the many-sided quality of request $\mathrm{O}(\mathrm{MK})$. Be that as it may, he cutting procedure of uplink MAC access and range has the many-sided quality of request $\mathrm{O}$ ( $\mathrm{K} \mathrm{K} \mathrm{fcsma/ca),} \mathrm{where} \mathrm{Kc}$ means the quantity of fighting UDs in the CSMA/CA outline part, fCSMA/CA. The SDN controller runs every time frame, $\triangle$, to reconfigure the asset portion to cuts and their particular UDs to ensure their objective downlink and uplink throughput, as appeared in Figure 6. Therefore the unpredictability of the proposed DAS2 plot for a cut $\mathrm{LiFi}$ attocell organize including $\mathrm{N}$ cut LiFi APs is roughly of request $\mathrm{O}(\mathrm{NMK} \triangle$ ). Various scalar tasks of request $\mathrm{O}(1)$ is additionally engaged with the procedure.

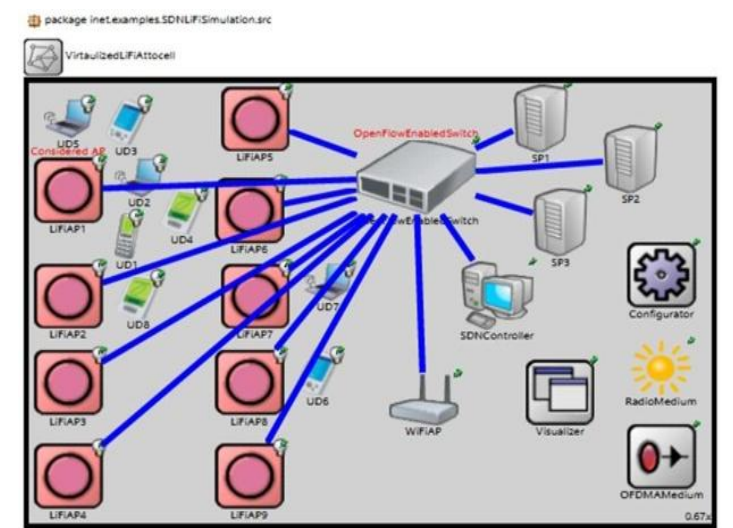

Figure 7. Virtualized LiFi attocell get to arrange recreation condition in $\mathrm{OMNeT++}$.

\section{SIMULATION AND RESULTS}

A reproduction situation has been produced in light of ns3 and utilizing OMNeT++ [19] to ponder the performance of the proposed DAS2 conspire for cutting downlink and uplink assets in $\mathrm{LiFi}$ attocell systems, as appeared in Figure7. In the reenactment setup, 9 LiFi APs are dispersed in the indoor Optima lab room of size $14 \times 6 \times 4(\mathrm{~W} \times \mathrm{L} \times$ $\mathrm{H}$ ), at the University-of-Edinburgh (UoE). The downlink what's more, uplink physical information rates of the LiFi $\mathrm{AP}$ are taken to be $19 \mathrm{Mbps}$, individually. The channel picks up are created in view of the Lambert and display portrayed in. The model thinks about every flag reflection from the distinctive dividers. The principle parameters of the LiFi AP are outlined in Table 1, and in Section III with a half-force radiation point $\varphi 1 / 2=60 \mathrm{o}$ and add up to number of OFDMA subcarriers, Nsc $=1024$. Three SPs are considered to share the assets in the $\mathrm{LiFi}$ attocell get to organize appeared in Figure 7.

Each SP has an application running on a cut in each of the distinctive LiFi APs to give administrations to their bought in UDs. The administration applications create downlink and uplink variable-bit rate (VBR), constant-bit rate (CBR), and best effort (BE) activity streams with normal information rates of $10 \mathrm{Mbps}$, 5Mbps, separately. The UDs and IoT gadgets (IoTDs) are dispersed over the distinctive 
attocell, with a higher thickness around the LiFi AP1 appeared in Figure 7. The parcel size of VBR, CBR and BE activity is taken to be $500,80,1500$ bytes, individually. Various execution assessment situations have been led to exhibit the abilities of the DAS2 plan to help asset cutting/virtualization in $\mathrm{LiFi}$ attocell systems. In this examination contemplate; the directed reproduction investigation centers around assessing the execution of the DAS2 plot in the LiFi AP1 appeared in Figure 7.

\subsection{Dynamic reasonable cut range assignment:}

This first scenario evaluates the ability of dynamic reasonable RBs (subcarriers) distribution to dynamic cuts in view of their deliberate activity stack. A functioning cut has movement to transmit or process for UDs.

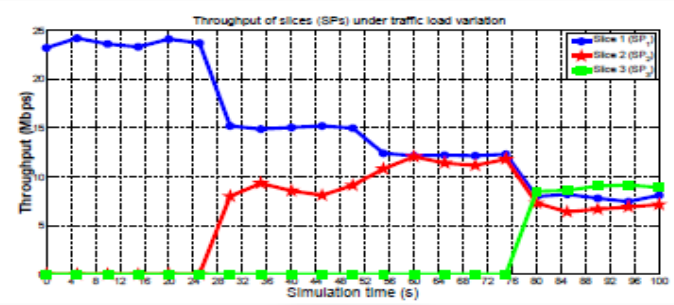

Figure 8. Throughput of slices under dynamic traffic load variations

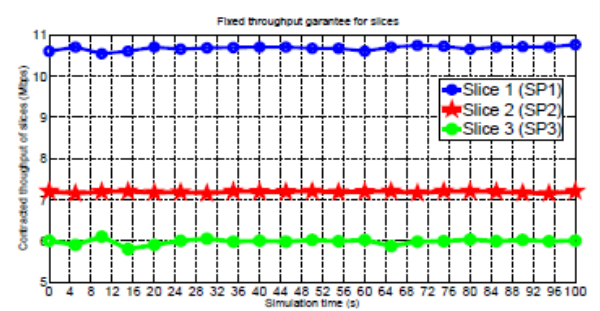

Figure. 9. Contracted throughput of slices ( S1 (45\%), S2 $(30 \%)$, S3 (25\%)).

The slices start to transmit different and 14 equal amounts of load at different times during the simulation. As a result, Fig. 8 shows that the slice (S1) is allocated almost the full downlink spectrum, when there is no traffic generated in the slices $2,3(\mathrm{~S} 2, \mathrm{~S} 3)$ from $\mathrm{t}=0$ to $\mathrm{t}=30 \mathrm{~s}$. When, however, $\mathrm{S} 2$ starts to generate traffic at $\mathrm{t}=30$, the full spectrum is gradually allocated in a proportional and fair manner among active $\mathrm{S} 1,2$ until $\mathrm{t}=55$. This trend of gradual dynamic fair spectrum allocation among slices continues, as generated traffic in S3 increases at $\mathrm{t}=75$. At this time, the OThroughput of slices under different BE traffic loads different slices (S1, 2, 3) generate an almost equal traffic load and therefore they equally share the full spectrum. Thus, the downlink virtualization scheme could adjust the transmission rate of active slices based on their generated traffic load to guarantee fair throughput among them. This demonstrates that the proposed scheme ensures statistical resource multiplexing. It distributes the unused resources of slices with light load among the slices with high load. This increases, in turn, the total throughput of the LiFi AP, while ensuring the performance fairness among all the supported active slices.

\subsection{Fixed slice spectrum guarantee:}

This second scenario assesses the capability of the DAS2 scheme to guarantee the target downlink throughput level of $\mathrm{S} 1, \mathrm{~S} 2$, and S3 agreed in the SLA with the ISP. S1, S2, S3 are guaranteed $45 \%, 30 \%, 25 \%$, of the effective downlink spectrum, respectively. The UDs move a half meter per second among the different attocell toward the marked $\mathrm{LiFi}$ AP1 in the network shown in Figure 7. As a result, Fig. 9 shows that the required resources to achieve the contracted target throughput levels are guaranteed for each active slice. This enables SPs to provision guaranteed services for UDs with a guaranteed data rate level. Despite the UDs' mobility among the different attocell, the DAS2 scheme maintains the throughput of the different slices.

This demonstrates that the slices are completely isolated and traffic class and volume in one slice do not influence the throughput performance of other slices.

\subsection{Minimum slice spectrum guarantee:}

This third scenario assesses the capability of allocating a minimum through put to each slice based on a measured traffic class of the supported application, irrespective of the heavily generated best effort (BE) background traffic in a $\mathrm{LiFi}$ attocell. The minimum throughout (data rate) of slices $\mathrm{S} 1, \mathrm{~S} 2, \mathrm{~S} 3$ is taken to be $6 \mathrm{Mbps}$. The slices, S1, S2, S3, support guaranteed bandwidth application (NG-VBR), nonguaranteed bandwidth application. ( $\mathrm{N}$ tt $-\mathrm{V}$ BR) and constant bit-rate application (CBR), respectively. The background BE traffic is generated across the slices using Pareto distribution with the Hurst parameter gradually increasing in this interval $\mathrm{H}=[0.54,0.89]$. As a result, Figure 10 shows that the DAS2 scheme guarantees a minimum throughput for $\mathrm{S} 1,3$, which is required to

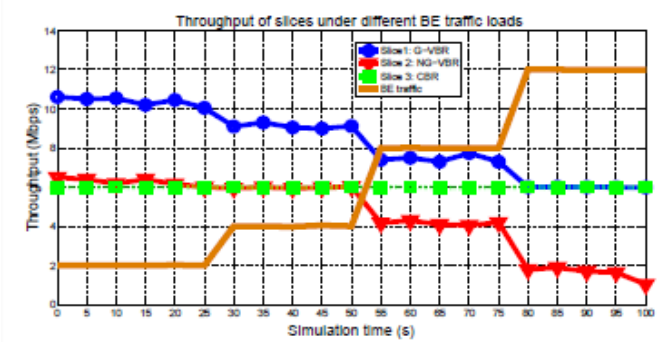

\section{Figure10. Throughput of slices under different BE traffic loads}

Support different traffic classes independent of BE traffic volume. When the BE traffic exceeds a specific volume level, the Ntt - V BR traffic rate generated on S2 falls below the minimum guaranteed throughput. This result demonstrates the control ability of DAS2 to maintain the resource ratio to the target throughput ratio of the different slices and applications providing guaranteed services to end UDs.

\subsection{Slice average delay guarantee:}

This scenario assesses the capability of the DAS2 scheme to support delay constrained applications on virtualized $\mathrm{LiFi}$ AP slices. The slices S1,2,3 are configured to support VoIP 


\section{AN ENHANCED ON BIDIRECTIONAL LI-FI ATTOCELL ACCESS POINT SLICING AND VIRTUALIZATION USING DAS2 CONSPIRE}

application, guaranteed bandwidth (G-VBR) video traffic and BE traffic, with a maximum delay constraint of $20 \mathrm{~ms}$ and $40 \mathrm{~ms}$ for $\mathrm{S} 1,2$, respectively, as well as an unlimited delay upper bound for S3, respectively. As a result, Figure 11 shows that the average delay of constrained traffic packets in each slice is guaranteed within the acceptable delay bounds of their application priority class. Generally, the achieved throughput and delay are based on the channel conditions of the LiFi UDs subscribed with each SP. However, the proposed DAS2 scheme guarantees the delay for each slice's SP based on the generated traffic class of supported applications. The obtained results show an elastic and rigorous control of LiFi downlink resources allocation for instantiated slices in the LiFi AP1 based on their measured traffic and QoS enforcement modules.

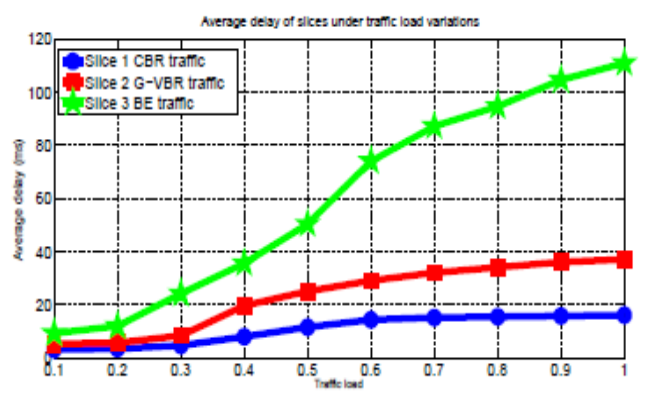

Figure 11. Normal postponement of cuts under various movement loads

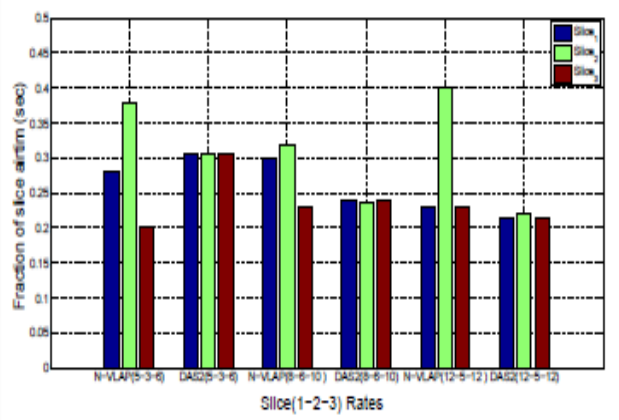

Figure12. Partial of cut broadcast appointment under various physical transmission rates

Movement and QoS requirement modules. This makes it conceivable to share assets among the bolstered cuts in view of QoS imperatives to accomplish particular business targets. This empowers, thusly, the ISPs to adequately allot $\mathrm{LiFi}$ foundation assets to the distinctive SPs to expand their income and differentiate their administrations provisioning. Presently, we examine the aftereffects of the directed situations to ponder the execution of the DAS2 plot in virtual zing LiFi AP uplink asset. Different quantities of dynamic UDs are bought in with the distinctive cuts characterized in each LiFi AP. Notwithstanding, our investigation is centered around the execution of UDs may produce diverse movement classes. Jain Fair's Index (JFI), is utilized to assess the UL broadcast appointment decency crosswise over uplink streams and conceded streams inside cuts. JFI measures the varieties of UL divert time use in and crosswise over cuts, which is given as takes after:
The airtime utilization of UDs in the different slices is inversely related to their physical data transmission rates and least blockage window, $\mathrm{C} \mathrm{W}$ min, allotted to them amid the MAC get to process. The produced movement is formed at the UDs and acclimated at the LiFi AP to give relative reasonable channel use among the UDs inside and across their cuts. The DAS2 plot distributes diverse cut weights to relatively shift the broadcast appointment among the dynamic UDs inside their cut.

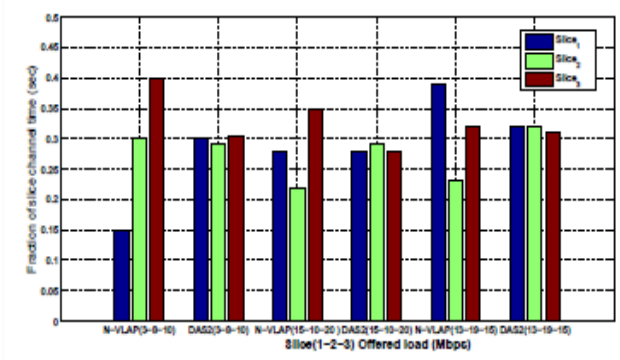

Figure 13. Fragmentary of cut broadcast appointment under changing offered activity stack.

The DAS2 plot distributes diverse cut weights to relatively shift the broadcast appointment among the dynamic UDs inside their cut. This permits, thusly, reasonable co where ri means the entirety of the channel time part utilized by all UDs to transmit uplink movement in cut $\mathrm{m}$; and $\mathrm{M}$ signifies the quantity of cuts. The execution of our proposed DAS2 in virtual zing LiFi AP uplink assets is contrasted with a non-virtualized LiFi AP (N-VLAP) running the same talked about up-connect mixture MAC convention. The accompanying subsections talk about the diverse directed situations to assess LiFi AP uplink assets cutting.

\subsection{Varying uplink physical transmission rate:}

This first uplink assets virtualization situation assesses the effect of shifting the qualities of movement created by dynamic UDs on the broadcast appointment proportion of their cuts. Figure 12 analyzes the partial channel time of cuts which have UDs transmitting at various physical transmission rates. This exhibits the DAS2 plan can relatively control broadcast appointment assignment among UDs inside and crosswise over cuts. The DAS2 plan could ensure an equivalent level of broadcast appointment sharing among cuts, contrasting with a N-VLAP. This neglected to ensure the broadcast appointment usage among the UDs in the cuts and among the diverse cuts. The broadcast appointment use of UDs in the distinctive cuts is conversely identified with their physical information transmission presence of transport conventions with various prerequisites.

\subsection{Varying uplink offered stack:}

This situation thinks about the partial channel time of cuts under various uplink movement loads produced by dynamic UDs in the diverse cuts instantiated in each LiFi AP. The DAS2 plan could ensure an equivalent offer of broadcast appointment among the bolstered cuts in the LiFi AP, as

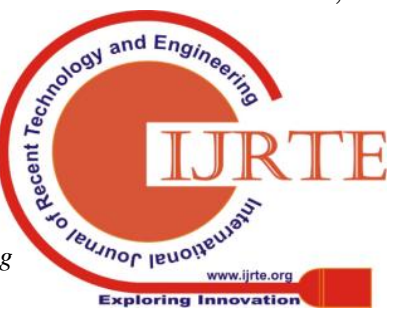


appeared in Figure 13. Notwithstanding, there is a divergence in the usage of the fragmentary divert broadcast appointment in the N-VLAP. The parcel measure varieties affect the general sharing of broadcast appointment at the LiFi AP for the UDs in the diverse cuts. Without the DAS2 conspire the outcome demonstrates that the broadcast appointment use at the AP specifically relies upon the span of the uplink movement bundles created by the dynamic UDs in the diverse cuts. The DAS2 conspire controls uplink activity by allotting relative broadcast appointment utilization among the UDs in the distinctive cuts. This relies upon parcel factual multiplexing broadcasting live interface of UDs. The DAS2 conspire empowers the control of broadcast appointment among the UDs in the diverse cuts.

\subsection{Uplink range reasonableness sharing:}

This situation com-pares the decency in uplink broadcast appointment sharing among the UDs inside and crosswise over cuts instantiated in a LiFi AP utilizing the DAS2 plot for the same UDs accepting administrations in the N-VLAP. The adjusted JFI metric in Eq.(23) is utilized APs. It additionally distributes assets to dynamic cuts in a dynamic and autonomic way. The downlink and uplink assets are viably shared among the diverse cuts instantiated in the LiFi attocell APs. The DAS2 plot has broadened the half and half TDMAS/CSMA/CA MAC convention and the worldwide token bank to help $\mathrm{LiFi}$ uplink MAC access and range virtualization. The SDN controller gives the essential data criticism to influence the DAS2 to plot activity class mindful and in this manner distributes automically the assets to the distinctive dynamic cuts. The downlink and uplink movement bundles to process the broadcast appointment sharing reasonableness and varieties.

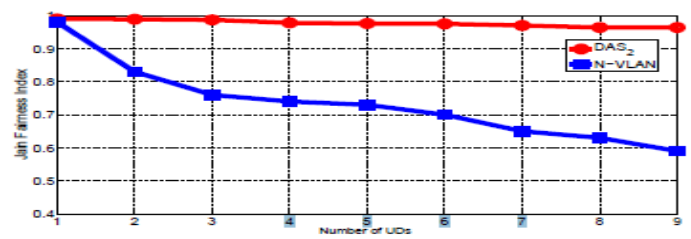

\section{Figure14. Broadcast appointment reasonableness correlations of DAS2 and N-VLAP.}

The ability of the DAS2 plan to guarantee corresponding reasonableness empowers an express control of the range proportions among the UDs given adequate accessible aggregate power transmission [17]. Therefore, Figure 14 demonstrates that the genuine broadcast appointment is nearly the same as the objective for various estimations of the base clog window, $\mathrm{C} \mathrm{W}$ min. This outcome shows the capacity of the DAS2 plan to ensure reasonableness among the upheld cuts contrasted with the N-VLAP. The proposed DAS2 conspire keeps up the intra/entomb cut reasonableness list JFI more noteworthy than 0.97, while the decency list tumbles down to 0.6 in the N-VLAP. The DAS2 plot powerfully measures the broadcast appointment for each cut and adjusts its assets, which result in a more steady decency execution. It can't achieve the full channel limit, yet it can share the rest of the broadcast appointment decently.

\section{DISCUSSION}

This situation looks at the effect of the DAS2 plot on the normal throughput of the LiFi uplink channel under an alternate number of UDs to the throughput of N-VLAP. Therefore, Figure 15 demonstrates that the DAS2 conspire accomplishes a superior throughput execution than the normal throughput in N-VLAP. The DAS2 plan can all the more likely use the uplink range of the diverse cuts by utilizing measurable multiplexing. This clarifies the expansion in the deliberate LiFi uplink throughput.

\section{CONCLUSION}

This paper has acquainted the DAS2 conspire with help asset virtualization and cutting in $\mathrm{LiFi}$ attocell get to networks. It bolsters uplink and downlink asset cutting of LiFi are overseen by the nearby LiFi AP asset director. This keeps up the objective throughput and postponement of the distinctive bolstered activity classes on the cuts upheld in each $\mathrm{LiFi} \mathrm{AP}$ in the LiFi attocell arrange. The got comes about demonstrate a critical change in the sharing of the downlink assets and uplink broadcast appointment decency, while keeping up the throughput level of the upheld cuts in each LiFi AP. The IEEE 802.11 circulated control work (DCF) progressively adjusts the base dispute window of fighting UDs to keep up broadcast appointment reasonableness. The acquired outcomes exhibit the ability of DAS2 to help a self-assertive number of cuts in a LiFi AP while ensuring the QoS prerequisites of uses running on the diverse cuts. The DAS2 plan can be incorporated into any LiFi AP to help various administrations, which can offer separated nature of-encounter $(\mathrm{QoE})$ for end clients while utilizing LiFi assets in a productive, viable and segregated way. The directed research contemplate speaks to the initial step to show the possibility of the created DAS2 plan to help an autonomic remote asset virtualization design for virtual zing $\mathrm{LiFi}$ attocell get to systems. These qualities recognize the DAS2 plot from the other proposed cutting and virtualization approaches [18]. The proposed plot requires assist improvement to help numerous investigations to keep running on the same LiFi attocell organize, while ensuring the exercises of trials running on the diverse cuts detached from each other. The subsequent stage in this examination study will create dispersed asset virtualization calculations that can all the more likely improve asset partaking in $\mathrm{LiFi}$ attocell get to systems.

\section{ACKNOWLEDGEMENT:}

Authors would like to express sincere gratitude to management and principal of S R Engineering College, for their support and encouragement to carry out the research work. 

USING DAS2 CONSPIRE

\section{REFERENCES:}

1. A. S. Thyagaturu, A. Mercian, M. P. McGarry, M. Reisslein, W. Kellerer, "Software defined optical networks (SDONs): A comprehensive survey", IEEE Commun. Surveys Tuts., vol. 18, no. 4, pp. 2738-2786, 4th Quart. 2016.

2. A. Blenk, A. Basta, M. Reisslein, W. Kellerer, "Survey on network virtualization hypervisors for software defined networking", IEEE Commun. Surveys Tuts., vol. 18, no. 1, pp. 655-685, 1st Quart. 2016.

3. K. Samdanis, X. Costa-Perez, V. Sciancalepore, "From network sharing to multi-tenancy: The $5 \mathrm{G}$ network slice broker", IEEE Commun. Mag., vol. 54, no. 7, pp. 32-39, Jul. 2016.

4. M. Richart, J. Baliosian, J. Serrat, J. L. Gorricho, "Resource slicing in virtual wireless networks: A survey", IEEE Trans. Netw. Service Manag., vol. 13, no. 3, pp. 462-476, Sep. 2016.

5. M. Zeng et al., "Design and demonstration of an indoor visible light communication network with dynamic user access and resource allocation", Proc. 9th Int. Conf. Wireless Commun. Signal Process. (WCSP), pp. 2472-7628, Dec. 2017.

6. M. Sheshikala, D. Rajeswara Rao, and R. Vijaya Prakash "Computation Analysis for Finding Co-Location Patterns using Map-Reduce Framework", Indian Journal of Science and Technology, Volume 10, Issue 7, Feb 2017, pp 1-6, ISSN: 0974-6846.

7. D. Tsonev, S. Sinanovic, and H. Haas, "Practical MIMO Capacity for Indoor Optical Wireless Communication with White LEDs," in Proc. of IEEE VTC-Spring, June 2013.

8. H. Alshaer, "An overview of network virtualization and cloud network as a service," International Journal of Network Management, vol. 25, no. 1, pp. 1-30, January/February 2015.

9. Ashish, S.Naresh Kumar, "Legitimate Traffic Jamming Prevention", Journal of Engineering Research and Applications, Volume 3, Issue 6, November 2013. Pp 682688, ISSN: 2248-9622.

10. Z. Chen, N. Serafimovski, and H. Haas, "Angle Diversity for an Indoor Cellular Visible Light Communication System," in Proc. of IEEE 2014 Vehicular Technology Conference (VTC Spring), Seoul, Korea, December 2014, pp. 1-5.

11. B. Shrestha, E. Hossain, and K. W. Choi, "Distributed and Centralized Hybrid CSMA/CA-TDMA Schemes for Singlehop Wireless Networks," IEEE Journal in Selected Areas Communications, vol. 13, no. 7, pp. 4050-4065, July 2014

12. Y. Khan, M. Derakhshani, S. Parsaeefard, and T. L. Ngoc, "Self-organizing TDMA MAC Protocol for Effective Capacity Improvement in IEEE 802.11 WLANs," in Proc. of IEEE Globecom Workshops (GC Wkshps), December 2015, p. 16.

13. B. Shrestha, K. W. Choi, and E. Hossain, "A dynamic time slot allocation scheme for hybrid CSMA/TDMA MAC protocol," IEEE Wireless Communications Letter, vol. 2, no. 5, pp. 535-538, October 2013.

14. DeepikaVodnala, S.Phani Kumar, Srinivas Aluvala, "An Efficient On-demand Link Failure Local Recovery Multicast Routing Protocol",Advances in Intelligent Systems and Computing, Volume 467, Issue 1, Oct 2016, pp 603-616, ISBN: 978-981-10-1644-8.

15. R. Kokku, R. Mahindra, H. Zhang, and S. Rangarajan, "NVS: A Virtualization Substrate for WiMAX Networks," in Proc.of MobiCom '10, Chicago, Illinois, USA, September 2010, pp. 233-244.

16. T. Guo and R. Arnott, "Active LTE RAN Sharing with Partial Resource Reservation," in Proc.of IEEE 78th Vehicular Technology Conference (VTC Fall), Las Vegas, NV, USA, September 2013, pp. 1-5.

17. R. Kokku, R. Mahindra, H. Zhang, and S. Rangarajan, "NVS: A substrate for virtualizing wireless resources in cellular networks," IEEE/ACM Transactions on Networking, vol. 20, pp. 1333-1346, October 2012.

18. L. S. Puthalath, J. S.Zander, R. Merz, A. Feldmann, and T. Vazao, "Towards programmable enterprise WLANS with
Odin," in Proc. of HotSDN '12, Helsinki,Finland, August 2012, pp. 115-120.

19. OMNeT++, https://omnetpp.org/.

\section{AUTHORS PROFILE:}

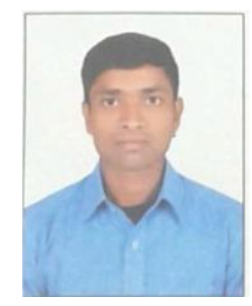

Yerrolla Chanti received Master's degree in Computer Science and Engineering in 2016 from Jawaharlal Nehru Technological University, Hyderabad, India. He has the teaching experience of 3 years. Currently, he is working as Associate Professor in Computer Science and Engineering Department at S R Engineering College, Warangal, India. His research areas include Networking, Big Data Analytics. 